\title{
WORKING GROUP FOR PLANETARY SYSTEM NOMENCLATURE (WGPSN) (GROUP DE TRAVAIL POUR LA NOMENCLATURE DU SYSTEM PLANETAIRE)
}

\author{
PRESIDENT: K. Aksnes \\ MEMBERS: $\quad$ M. Ya. Marov, B. G. Marsden, P. Moore, D. Morrison, \\ T. C. Owen, V. V. Shevchenko, B. A. Smith
}

CONSUltants: G. A. Burba, L. Gaddis, P. Masson, J. Blue

\section{Introduction}

Since the 22nd IAU General Assembly in the Hague in 1994, WGPSN has conducted most of its business by e-mail and fax. Ad hoc WG meetings were held on July 10, 1996 in Versailles and on July 17 in Birmingham during, respectively, the Asteroids, Comets and Meteors meeting and the 31st COSPAR Scientific Assembly. Seven members of the WG and two members of the adhering Nomenclature Task Groups attended one of the nomenclature meetings or both.

During the triennium most of the nomenclature work has been concerned with classifying and naming features on Venus and the asteroids Gaspra and Ida as observed by the U.S. spacecraft Magellan and Galileo.

\subsection{MEMBERSHIP CHANGES}

We have been saddened by the tragic death of Stanley Keith Runcorn (1922-1995), professor at the University of Newcastle upon Tyne, who was killed in San Diego on December 5, 1995. Runcorn has been a member of the Task Group for Lunar Nomenclature ever since WGPSN was formed in 1973. He was a highly regarded geophysicist who has made many important contributions especially to the study of the Earth's magnetism and continental drift.

Joel Russell has retired from the U.S. Geological Survey in Flagstaff and has been replaced by Jennifer Blue at the same institution as a nomenclature coordinator and a consulting member of WGPSN.

\section{Nomenclature Corrections}

Re-inspection of Magellan radar images of Venus has made it necessary to make the following corrections to the Venus nomenclature earlier approved by the IAU (see also the table in the next section):

1. In IAU Trans. XIXB, 1985, omit Brigit Tholus and change Aspasia Patera, Cotis Mons, Furki Mons, and Mentha Mons to, respectively, Aspasia Corona, Cotis Tholus, Furki Tholus, and Mentha Tholus.

2. In IAU Trans. XXIA, 1991, change Cassatt Patera, Nzingha Patera, Schumann-Heink Patera, and Tituba Patera to, respectively, Cassatt Corona, Nzingha Corona, Schumann-Heink Corona, and Tituba Corona.

3. In IAU Trans. XXIIA, 1994, omit Montagu Crater and change Anala Corona and Somagalags Corona to, respectively, Anala Mons and Somagalags Montes.

4. In IAU Trans. XXIIB, 1994, omit Blanche Crater and Colonna Crater, change Graham Crater to Graham Patera, and change Lida Crater to Amanda Crater. 
In the nomenclature for Mars and Moon, the following corrections are needed:

1. In IAU Trans. XXIIA, 1994, change Marti Vallis to Marte Vallis.

2. In IAU Trans. XXIIB, 1994, omit reference to von Braun under Mars nomenclature and change spelling of von Braun's first name from Werner to Wernher under Lunar nomenclature.

\title{
3. New Nomenclature
}

\author{
E U R O P A
}

$\begin{array}{lccrc}\text { NAME } & \text { LAT } & \text { LONG } & \begin{array}{l}\text { DIAM } \\ (\mathrm{km})\end{array} & \text { ATTRIBUTE } \\ \begin{array}{l}\text { MACULA } \\ \text { Boeotia Macula }\end{array} & 54.0 \mathrm{~S} & 166.0 \mathrm{~W} & 22 & \begin{array}{l}\text { Place where Cadmus led cow before it } \\ \text { stopped at site of Thebes. }\end{array} \\ \begin{array}{l}\text { Cyclades Macula } \\ \text { Islands where Rhadamanthys reigned. }\end{array} & 64.0 \mathrm{~S} & 192.0 \mathrm{~W} & 105 & \\ \begin{array}{l}\text { LINEA } \\ \text { Astypalaea Linea }\end{array} & 76.5 \mathrm{~S} & 220.3 \mathrm{~W} & 1030 & \text { Sister of Europa. }\end{array}$

I D A

\section{CRATERS}

Atea

Azzurra

Bilemot

Castellana

Choukoutien

Fingal

Kartchner

Kazumura

Lascaux

Lechuguilla

Mammoth

Manjang

Orgnac

Padirac

Postojna

Sterkfontein

Stiffe

Undara

Viento

DORSUM

Townsend Dorsum
5.7S $18.9 \mathrm{E}$

$30.5 \mathrm{~N} \quad 217.2 \mathrm{E}$

$27.8 \mathrm{~S} \quad 29.2 \mathrm{E}$

13.4S $335.2 \mathrm{E}$

$12.8 \mathrm{~N} \quad 23.6 \mathrm{E}$

13.2S $39.9 \mathrm{E}$

7.OS $179.0 \mathrm{E}$

32. OS $41.1 \mathrm{E}$

$0.8 \mathrm{~N} \quad 161.2 \mathrm{E}$

$7.9 \mathrm{~N} \quad 357.1 \mathrm{E}$

18. 3

$180.3 \mathrm{E}$

$28.3 \mathrm{~S}$

$90.5 \mathrm{E}$

$6.3 \mathrm{~S}$

$202.7 \mathrm{E}$

$4.3 \mathrm{~S}$

$42.9 \mathrm{~S}$

5. $2 \mathrm{E}$

$4.1 \mathrm{~S}$

$359.9 \mathrm{E}$

27.95

$54.1 \mathrm{E}$

$113.8 \mathrm{E}$

12. $2 \mathrm{~N} \quad 343.9 \mathrm{E}$

$25.0 N$
$30.0 \mathrm{O} \quad 40$
2 Cave in the Muller Range of Papua, New Guinea.

9.6 Flooded cave in Southern Italy known as the Blue Grotto.

1.8 Lava tube in Korea.

5.2 Cave in Italy.

1.1 Site where Peking Man was discovered.

1.5 Cave in the Hebrides.

0.9 Cave in Arizona.

2.1 Lava tube in Hawaii.

11.8 Cave in France noted for its prehistoric paintings.

1.5 Cave in Carlsbad National Park, New Mexico.

10.2 Limestone cavern in Kentucky, U.S.A., known as the longest on Earth.

1 Lava tube in Korea.

10.6 Cave in France.

1.9 Cave with underground river in France.

6 Large cave in Slovenia.

4.7 Cave in South. Africa.

1.5 Karst cave in Sulmona, Italy.

8.5 Lava tube from Undara Volcano, North Queensland, Australia.

1.6 Lava tube in Spain.

Tim E. (d. 1989); Galileo imaging team member. 
I D A (cont.)

$\begin{array}{lcccc}\text { NAME } & \text { LAT } & \text { LONG } & \begin{array}{l}\text { DIAM } \\ (\mathrm{km})\end{array} & \text { ATTRIBUTE } \\ \begin{array}{l}\text { REGIO } \\ \text { Palisa Regio }\end{array} & 23.0 \mathrm{~S} & 34.0 \mathrm{E} & 23 & \begin{array}{l}\text { Johann }(1848-1925) ; \text { Austrian } \\ \text { astronomer, discovered Ida. }\end{array} \\ \text { Pola Regio } & 11.0 \mathrm{~S} & 184.0 \mathrm{E} & 8 & \begin{array}{l}\text { Place where Palisa (discoverer } \\ \text { of Ida) observed. } \\ \text { Where Palisa discovered Ida. }\end{array} \\ \text { Vienna Regio } & 8.0 \mathrm{~N} & 2.0 \mathrm{E} & 13 & \end{array}$

D A C T Y L

CRATERS

Acmon

Celmis

NAME

CRATERS

Ahava

Amanda

Batya

Bender

Bernice

Deborah

Dorothy

Elizabeth

Ellen

Enid

Evelyn

Ezraela

Georgina

Jane

Karen

Kelila

Kristina

Lara

Leslie.

Linda

Magdalena

Marianne

Melina

Mildred

Nadia

Nicole
$39.0 \mathrm{~S} \quad 138 . \mathrm{OE}$

$46.0 \mathrm{~S} \quad 220.0 \mathrm{E}$

VENUS

LAT LONG

DIAM

ATTRIBUTE

(km)

53.6N 187.3E 9 Hebrew first name.

29.1S 94.5E 13 Latin first name. Name changed from Lida.

72.8N 235.5E 7 Hebrew first name.

12.9S 327.3E 38 Heidi Julia (1979-1991); author and artist.

40.7S 14.8E 12 Greek first name.

37.3S 10.6E 8 Hebrew first name.

35.4S 10.9E 6 Greek first name.

59.2N 215.4E 11 Hebrew first name.

22.7S 281.3E 15 German first name.

16.4N 352.2E 7 Celtic first name.

61.OS 212.3E 18 Celtic first name.

57.0N 186.8E 9 Hebrew first name.

20.3S 58.6E 5 Greek first name.

60.4S $304.8 \mathrm{E} \quad 10$ Hebrew first name.

12.4S 17.7E 11 Greek first name.

$52.6 \mathrm{~N} \quad 191.8 \mathrm{E} \quad 6$ Hebrew first name.

65.2S 315.9E 9 Slavonic form of Christiana, Latin first name.

4.2S 2.9E 4 Latin first name.

11.2S 13.5E 7. English first name.

12.4S 2.7E 8 Latin first name.

11.2S 48.7E 10 Spanish form of Hebrew first name.

9.2N 358.OE 7 From Mary, Greek first name.

69.9S 319.5E 9 Greek first name.

51.7S 348.3E 11 English first name.

27.9S $\quad 0.6 \mathrm{E} \quad 10$ Russian first name.

48.4N 259.2E 8 French first name. 
VEN U S (cont.)

\begin{tabular}{|c|c|c|c|c|}
\hline NAME & LAT & LONG & $\begin{array}{l}\text { DIAM } \\
(\mathrm{km})\end{array}$ & ATTRIBUTE \\
\hline \multicolumn{5}{|l|}{ CRATERS } \\
\hline Oksana & $11.9 \mathrm{~N}$ & $352 . \mathrm{OE}$ & 10 & Ukrainian first name. \\
\hline Paige & $1.2 S$ & $24.6 \mathrm{E}$ & 6 & Italian first name. \\
\hline Philomena & $40.7 \mathrm{~S}$ & $151.8 \mathrm{E}$ & 13 & Greek first name. \\
\hline Quimby & $5.7 \mathrm{~S}$ & $76.7 \mathrm{E}$ & 23 & Harriet (1884-1912); American aviatrix \\
\hline Rebecca & $12.1 \mathrm{~S}$ & $5.3 \mathrm{E}$ & 9 & Hebrew first name. \\
\hline Sandi & $68.1 \mathrm{~S}$ & $315.1 \mathrm{E}$ & 9 & From Alexandra, Greek first name. \\
\hline Tiffany & 8.75 & $22.8 \mathrm{E}$ & 6 & Greek first name. \\
\hline Valerie & $6.4 S$ & $31 . \mathrm{OE}$ & 10 & French first name. \\
\hline Vanessa & $5.9 \mathrm{~S}$ & $1.9 \mathrm{E}$ & 9 & Greek first name. \\
\hline Yetta & $58.6 \mathrm{~N}$ & $185.4 \mathrm{E}$ & 8 & From Henrietta, German first name. \\
\hline \multicolumn{5}{|l|}{ CHASMA } \\
\hline Latona Chasma & $26.0 \mathrm{~N}$ & $267.5 \mathrm{E}$ & 530 & Roman moon goddess. \\
\hline \multicolumn{5}{|l|}{ COLLES } \\
\hline Olosa Colles & $18.0 \mathrm{~N}$ & $353.3 \mathrm{E}$ & 200 & Yoruba (Nigeria) lagoon goddess. \\
\hline \multicolumn{5}{|l|}{ CORONA } \\
\hline Aspasia Corona & $56.1 \mathrm{~N}$ & $189.1 \mathrm{E}$ & 200 & $\begin{array}{l}\text { Outstanding Greek woman (ca } 470-429 \\
\text { B.C.). Changed from Aspasia Patera. }\end{array}$ \\
\hline Banba Corona & $47.2 \mathrm{~S}$ & $209.2 \mathrm{E}$ & 110 & Irish earth goddess. \\
\hline Cassatt Corona & $65.6 \mathrm{~N}$ & $207.6 \mathrm{E}$ & 152 & $\begin{array}{l}\text { Mary (1844-1926); American painter. } \\
\text { Changed from Cassatt Patera. }\end{array}$ \\
\hline Cerridwen Corona & $49.6 \mathrm{~N}$ & $201.8 \mathrm{E}$ & 217 & Celtic nature goddess. \\
\hline Hulda Corona & $12.0 N$ & $308.3 \mathrm{E}$ & 230 & $\begin{array}{l}\text { German goddess of fruitfulness \& } \\
\text { marriage. }\end{array}$ \\
\hline Iyatik Corona & $16.5 \mathrm{~S}$ & $347.5 \mathrm{E}$ & 200 & $\begin{array}{l}\text { Keresan Pueblo (New Mexico) corn } \\
\text { mother. }\end{array}$ \\
\hline Ki Corona & $43.2 \mathrm{~N}$ & $227.8 \mathrm{E}$ & 300 & Sumerian earth goddess. \\
\hline Ma Corona & $22.5 \mathrm{~S}$ & $57.0 \mathrm{E}$ & 420 & Fertility goddess from Asia Minor. \\
\hline Madderakka Corona & $9.0 \mathrm{~N}$ & $315.5 \mathrm{E}$ & 220 & Lapp goddess of birth. \\
\hline Muzamuza Corona & $65.6 \mathrm{~N}$ & $205.4 \mathrm{E}$ & 163 & Indian earth goddess. \\
\hline Nalwanga Corona & $48.7 N$ & $247 . \mathrm{OE}$ & 380 & Ganda (Uganda) goddess of childbirth. \\
\hline Ninkarraka Corona & $65.3 \mathrm{~N}$ & $221 . \mathrm{OE}$ & 150 & Babylonian goddess of childbirth. \\
\hline Nott Corona & $32.3 S$ & 202. OE & 150 & Scandinavian earth goddess. \\
\hline Nzingha Corona & $68.7 N$ & $205.7 \mathrm{E}$ & 140 & $\begin{array}{l}\text { (Ann Zingha) (1582-1663), queen, head } \\
\text { of Amazon band. } \\
\text { Changed from Nzingha Patera. }\end{array}$ \\
\hline Pachamama Corona & $36.0 \mathrm{~S}$ & $21.8 \mathrm{E}$ & 130 & Incan earth goddess. \\
\hline $\begin{array}{l}\text { Schumann-Heink } \\
\text { Corona }\end{array}$ & $74.3 \mathrm{~N}$ & $214.5 \mathrm{E}$ & 122 & $\begin{array}{l}\text { Ernestine (1861-1963); German singer. } \\
\text { Changed from Schumann-Heink Patera. }\end{array}$ \\
\hline Silvia Corona & $12.6 \mathrm{~N}$ & $355.7 \mathrm{E}$ & 270 & Roman earth goddess. \\
\hline Tituba Corona & $42.4 \mathrm{~N}$ & $214.7 \mathrm{E}$ & 163 & $\begin{array}{l}\text { Nurse who started Salem witch hunt } \\
\text { (c. 1692). Changed from Tituba Patera. }\end{array}$ \\
\hline Corona & $31.5 \mathrm{~N}$ & $258.3 E$ & 315 & Persian earth goddess. \\
\hline
\end{tabular}


V E N U S (cont.)

\begin{tabular}{|c|c|c|c|c|}
\hline NAME & LAT & LONG & $\begin{array}{l}\text { DIAM } \\
(\mathrm{km})\end{array}$ & ATTRIBUTE \\
\hline \multicolumn{5}{|l|}{ DORSUM/DORSA } \\
\hline Aditi Dorsa & $30.0 \mathrm{~S}$ & $189.0 \mathrm{E}$ & 1200 & Indian sky goddess. \\
\hline Sirona Dorsa & $43.5 \mathrm{~S}$ & $193.5 \mathrm{E}$ & 700 & Celtic sky goddess. \\
\hline Surupa Dorsum & $71.7 \mathrm{~N}$ & $209.0 \mathrm{E}$ & 981 & Hindu sky goddess, bringer of rain. \\
\hline \multicolumn{5}{|l|}{ FLUCTUS } \\
\hline Nekhebet Fluctus & $0.0 \mathrm{~N}$ & $35.0 \mathrm{E}$ & 400 & Egyptian vulture goddess. \\
\hline Strenia Fluctus & $41.0 \mathrm{~N}$ & $250.0 \mathrm{E}$ & 490 & $\begin{array}{l}\text { Roman goddess of New Years } \\
\text { festivities. }\end{array}$ \\
\hline Ubastet Fluctus & $48.0 \mathrm{~S}$ & $27 . \mathrm{OE}$ & 550 & Egyptian cat goddess. \\
\hline Uzume Fluctus & $28.6 \mathrm{~N}$ & $220.3 \mathrm{E}$ & 700 & Japanese goddess of cheerfulness. \\
\hline Zipaltonal Fluctus & $37.7 \mathrm{~N}$ & $251.3 E$ & 290 & Nicaraguan creator goddess. \\
\hline \multicolumn{5}{|l|}{ FOSSAE } \\
\hline Fea Fossae & $27.5 \mathrm{~N}$ & $224 . \mathrm{OE}$ & 620 & Gaelic war goddess. \\
\hline \multicolumn{5}{|l|}{ LINEA } \\
\hline Virtus Linea & $12.0 \mathrm{~N}$ & $21 . \mathrm{OE}$ & 500 & ' Roman war goddess. \\
\hline \multicolumn{5}{|l|}{ MONS/MONTES } \\
\hline Anala Mons & $11.0 \mathrm{~N}$ & $14.1 \mathrm{E}$ & 525 & $\begin{array}{l}\text { Hindu fertility goddess. } \\
\text { Name changed from Anala Corona. }\end{array}$ \\
\hline Atanua Mons & $9.5 \mathrm{~N}$ & $308.9 \mathrm{E}$ & 1000 & Dawn goddess from Marquesas Islands. \\
\hline Irnini Mons & $14.6 \mathrm{~N}$ & 16. OE & 525 & Goddess of cedar-tree mountains. \\
\hline Kali Mons & $9.4 \mathrm{~N}$ & $29.2 \mathrm{E}$ & 325 & Hindu goddess, mother of death. \\
\hline Nyx Mons & $30.0 \mathrm{~N}$ & $48.5 \mathrm{E}$ & 875 & Greek goddess of night. \\
\hline Somagalags Montes & $9.3 \mathrm{~N}$ & $348.5 E$ & 105 & $\begin{array}{l}\text { Bella Coola earth mother. } \\
\text { Name changed from Somagalags Corona. }\end{array}$ \\
\hline Tuli Mons & $13.3 \mathrm{~N}$ & $314.6 \mathrm{E}$ & 750 & Samoan goddess of creation. \\
\hline Var Mons & $1.2 \mathrm{~N}$ & $316.2 \mathrm{E}$ & 1000 & Scandinavian love goddess. \\
\hline \multicolumn{5}{|l|}{ PATERA } \\
\hline Graham Patera & $6.0 \mathrm{~S}$ & $6.0 \mathrm{E}$ & 75 & $\begin{array}{l}\text { Martha (1894-1991); American dancer, } \\
\text { choreographer. } \\
\text { Changed from Graham Crater. }\end{array}$ \\
\hline Kupo Patera & $41.9 \mathrm{~S}$ & $195.5 \mathrm{E}$ & 100 & Irena (1929-1978); Israeli astronomer. \\
\hline Libby Patera & $34.5 \mathrm{~S}$ & $199.5 \mathrm{E}$ & 90 & $\begin{array}{l}\text { Leona Woods Marshall (1919-1986); } \\
\text { American chemist and physicist. }\end{array}$ \\
\hline $\begin{array}{l}\text { Payne-Gaposchkin } \\
\text { Patera }\end{array}$ & $25.5 S$ & $196.0 \mathrm{E}$ & 100 & $\begin{array}{l}\text { Cecilia Helena (1900-1979); American } \\
\text { astronomer. }\end{array}$ \\
\hline \multicolumn{5}{|l|}{ RUPES } \\
\hline Vaidilute Rupes & $43.5 \mathrm{~S}$ & $22.0 \mathrm{E}$ & 2000 & Lithuanian hearth goddess. \\
\hline \multicolumn{5}{|l|}{ TESSERA } \\
\hline Bathkol Tessera & $61.0 \mathrm{~N}$ & $200.0 \mathrm{E}$ & 1485 & Isreali goddess of fate. \\
\hline Manatum Tessera & 4. OS & $64.0 \mathrm{E}$ & 3800 & Semitic goddess of fate. \\
\hline Salus Tessera & $1.5 \mathrm{~S}$ & $48.5 \mathrm{E}$ & 850 & Roman health and prosperity goddess. \\
\hline Tyche Tessera & $44.0 \mathrm{~S}$ & $14.5 \mathrm{E}$ & 575 & Greek goddess of fate. \\
\hline
\end{tabular}


VEN U S (cont.)

NAME

THOLUS/THOLI

Cotis Tholus

Evaki Tholus

Furki Tholus

Mentha Tholus

Otafuku Tholi

Toci Tholus

Tursa Tholus

VALLIS

Banumbirr Vallis

LAT

LONG

DIAM

$(\mathrm{km})$

44.3N 233.0E

62 Thracian goddess, similar to Cybele. Changed from Cotis Mons.

$37.6 \mathrm{~N} \quad 342.2 \mathrm{E} \quad 200$ Amazonian sleep goddess.

$35.9 \mathrm{~N} 236.4 \mathrm{E} \quad 79$ Chechen and Ingus (Caucasus) goddess, wife of thunder god Sela. Changed from Furki Mons.

43.ON 237.3E 79 Roman goddess personifying human mind. Changed from Mentha Mons.

28.7N 46.3E 80 Japanese goddess of joyfulness.

$29.5 \mathrm{~N} 355.1 \mathrm{E} \quad 300$ Aztec earthquake goddess.

$35.4 \mathrm{~S} 205.2 \mathrm{E} \quad 75$ Italian goddess of terror.
$7.0 \mathrm{~S}$
4. OE
400 Arnhemland word for planet Venus. 